\title{
A New Two-Lane Lattice Model with the Consideration of the Driver's Self-Anticipation Current Difference Effect
}

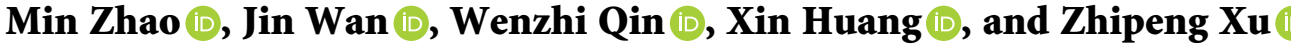 \\ School of Science, Nantong University, Jiangsu 226019, China \\ Correspondence should be addressed to Zhipeng Xu; xuzhp@ntu.edu.cn
}

Received 26 May 2021; Revised 8 November 2021; Accepted 29 December 2021; Published 24 January 2022

Academic Editor: M Syed Ali

Copyright (c) 2022 Min Zhao et al. This is an open access article distributed under the Creative Commons Attribution License, which permits unrestricted use, distribution, and reproduction in any medium, provided the original work is properly cited.

\begin{abstract}
To prevent traffic congestion, drivers always adjust the driving behavior with their driving information. By considering the selfanticipation effect and the optimal current difference effect on traffic flow stability, a novel two-lane lattice hydrodynamic model is proposed. Compared with Peng's model, the linear stability analysis results reveal that the self-anticipation term can effectively enlarge the stable region on the phase diagram. Then, a reductive perturbation method is used to derive the mKdV equation describing traffic congestion near the critical point. Nonlinear analyses show that the traffic congestions can be effectively suppressed by taking the coefficient of lane-changing behaviors $\gamma$ and the anticipation time $\tau$ into account. These results further indicate that the driver's self-anticipation current difference effect can efficiently alleviate traffic jams. Furthermore, the numerical simulations with periodic boundary conditions also confirm the effectiveness of theoretical results.
\end{abstract}

\section{Introduction}

With the rapid population expansion and car ownership growth, traffic congestion becomes a topic issue over the past decade. To solve this issue, various traffic models [1-7] have been put forward to investigate the characteristics of traffic flow. Generally, there are two kinds of traffic models, i.e., macroscopic traffic flow models [8-10] and microscopic traffic flow models [11-13]. The microscopic model focuses on the influence of individual vehicle behavior, including car-following models [14-19], cellular automaton models $[20,21]$, etc. While the macroscopic model usually uses flux, density, and mean speed to describe the dynamic behavior of traffic flow. Also, it can be divided into continuous models [22-24] and lattice hydrodynamic models [25, 26].

In 1998, Nagatani [27] firstly constructed the single-lane lattice dynamic model to describe the jamming transition and the density wave of traffic flow. Meanwhile, the kinkantikink soliton solutions of the $\mathrm{mKdV}$ equation were derived to depict the propagation of congested traffic. With the expanding scale of vehicles, the multilane highway has emerged to replace the single-lane highway, which greatly relieved the urban traffic pressure. Based on the single-lane case, Nagatani [28] developed a two-lane lattice hydrodynamic model to reveal the effect of lane changing on the stability of traffic flow. Since then, the two-lane lattice hydrodynamic model was extended with various traffic factors such as traffic flux difference [29-31], traffic density difference [32-34], optimal current difference [35-37], and anticipation effect $[38,39]$.

Anticipation effect, as a type of effective stabilization strategy for the traffic flow system, means that drivers can adjust their velocity according to the traffic conditions of preceding vehicles at the next moment [37]. In addition to the information from preceding vehicles, drivers always adjust the current vehicle's driving behavior with their driving information. Lately, Zhang et al. [40] studied the impact of the vehicle's self-anticipation density effect on traffic stability and proved that the traffic flow stability could be enhanced when the self-anticipation density effect was taken into account. Meanwhile, the optimal current difference between two successive lattices [35] has been proved to be effective in terms of traffic stability improvements. Through the above considerations, both the self-anticipation effect and the optimal current difference effect could contribute to the improvement of traffic flow stability. Up to 
now, little work has been performed for the optimal current difference with the self-anticipation effect. This motivates us to develop a novel two-lane lattice hydrodynamic model fully considering the optimal current difference with the selfanticipation effect and illustrate the effectiveness of this optimal current difference through theoretical analysis and numerical simulation.

The structure of this article is organized as follows. The extended model will be established in Section 2. The linear stability analysis of the model will be established in Section 3 . Section 4 will introduce the nonlinear analysis. Then, in Section 5, numerical simulations will be used to verify the previous theoretical analysis. Finally, the conclusions are summarized in Section 6.

\section{Model}

By incorporating the lane-changing behavior in multilane traffic problems, Nagatani [28] presented the original lattice hydrodynamic model for two-lane highway. Figure 1 shows the schematic model of the traffic flow system on the two-lane highway with the lane-changing behaviors. Let $\rho_{1, j}$ and $\rho_{2, j}$ denote the densities of two lanes, respectively. If $\rho_{m, j+1}(t)<\rho_{n, j}(t), m \neq n(m, n \in\{1,2\})$, then the lanechanging behavior appears from lane $n$ to lane $m$ at the rate $\gamma\left|\rho_{0}^{2} V^{\prime}\left(\rho_{0}\right)\right|\left(\rho_{n, j}(t)-\rho_{m, j+1}(t)\right)$. Here, $\gamma$ denotes a dimensionless fixed coefficient. Thus, one can get the continuity equations for two lanes:

$$
\begin{array}{r}
\partial_{t} \rho_{m, j}+\rho_{0}\left(\rho_{m, j} v_{m, j}-\rho_{m, j-1} v_{m, j-1}\right)=\gamma\left|\rho_{0}^{2} V^{\prime}\left(\rho_{0}\right)\right|\left(\rho_{n, j+1}-2 \rho_{m, j}+\rho_{n, j-1}\right), m \neq n(m, n \in\{1,2\}) . \\
\partial_{t}\left(\rho_{j} v_{j}\right)=a \rho_{0} V_{e}\left(\rho_{j+1}\right)-a \rho_{j} v_{j},
\end{array}
$$

Then, equation (1) can be reduced into

$\partial_{t} \rho_{j}+\rho_{0}\left(\rho_{j} v_{j}-\rho_{j-1} v_{j-1}\right)=\gamma\left|\rho_{0}^{2} V^{\prime}\left(\rho_{0}\right)\right|\left(\rho_{j+1}-2 \rho_{j}+\rho_{j-1}\right)$,

where $\rho_{j}=\rho_{1, j}+\rho_{2, j} / 2, \rho_{j} v_{j}=\rho_{1, j} v_{1, j}+\rho_{2, j} v_{2, j} / 2$. Accordingly, the evolution equation of a two-lane lattice hydrodynamic model was introduced as where $V_{e}\left(\rho_{j}\right)=V_{e}\left(\rho_{1, j}\right)+V_{e}\left(\rho_{2, j}\right) / 2$. To analyze the impact of the anticipation effect in traffic stabilization, Wang et al. [41] addressed a new single-lane lattice model described by

$$
\partial_{t}\left(\rho_{j}(t) v_{j}(t)\right)=a \rho_{0} V\left(\rho_{j+1}(t)+\beta\left(\rho_{j+1}(t+\tau)-\rho_{j+1}(t)\right)\right)-a \rho_{j}(t) v_{j}(t),
$$

where $\tau$ and $\beta$ represent the anticipation time and an influenced coefficient, respectively. $\rho_{j+1}(t+\tau)-\rho_{j+1}(t)$ is the predictive density variation at site $j+1$. In 2013, Peng
[35] constructed a novel two-lane lattice model with the optimal current difference effect

$$
\partial_{t}\left(\rho_{j}(t) v_{j}(t)\right)=a\left[\rho_{0} V\left(\rho_{j+1}(t)\right)-\rho_{j} v_{j}\right]+a \rho_{0} \lambda\left(V\left(\rho_{j+2}(t)\right)-V\left(\rho_{j+1}(t)\right)\right) \text {, }
$$

where $\lambda$ is the reaction coefficient and $\rho_{0}\left(V\left(\rho_{j+2}(t)\right)-V\left(\rho_{j+1}(t)\right)\right.$ represents the optimal current difference between two successive lattices $j+2$ and $j+1$ at time $t$.

As the essential factors in traffic flow discipline, both the driver's anticipation effect and the optimal current difference effect can moderate traffic congestion. Meanwhile, drivers are inclined to adjust their vehicles with their selfanticipation information rather than anticipation information from the successive lattices in real traffic scenarios.

In order to study the influence of driver's self-anticipation traffic information on traffic flow stability, a new evolution equation with the self-anticipation current difference effect (SCDE) is defined by

$$
\partial_{t}\left(\rho_{j} v_{j}\right)=a \rho_{0} V\left(\rho_{j+1}(t)\right)-a \rho_{j} v_{j}+a \rho_{0} \lambda V\left(\rho_{j+1}(t+\tau)\right)-V\left(\rho_{j+1}(t)\right) \text {, }
$$




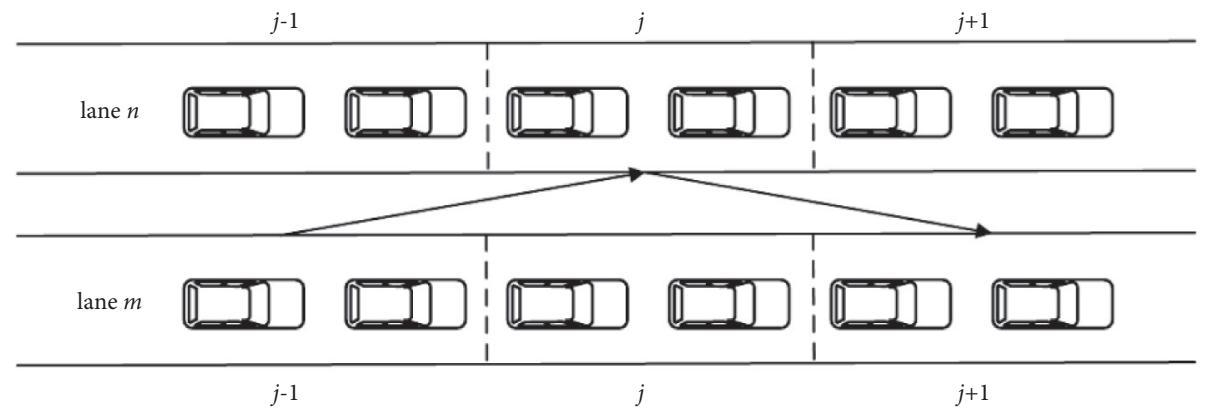

Figure 1: Schematic of the lattice hydrodynamic model.

where $\lambda$ is an anticipated response coefficient of current difference and $\rho_{0}\left(V\left(\rho_{j+1}(t+\tau)\right)-V\left(\rho_{j+1}(t)\right)\right)$ denotes the self-anticipation current difference of site $j+1$. In addition, the longer the time of $\tau$ is, the more attention the drivers would pay to their driving behavior, which tends to make traffic flow more stable.

For the sake of convenience, performing a Taylor expansion of variable $V\left(\rho_{j+1}(t+\tau)\right)$ and ignoring its nonlinear terms, equation (6) can be simplified as

$$
\partial_{t}\left(\rho_{j}(t) v_{j}(t)\right)=a\left[\rho_{0} V\left(\rho_{j+1}(t)\right)-\rho_{j}(t) v_{j}(t)\right]+a \rho_{0} \lambda\left(V^{\prime}\left(\rho_{j+1}(t)\right) \tau \partial_{t} \rho_{j+1}(t)\right) .
$$

By combing equation (6) with equation (2) and eliminating the velocity $\mathrm{v}_{j}$, the 2 -order density equation is included as

$$
\begin{gathered}
\partial_{t}^{2} \rho_{j}+a \rho_{0}^{2}\left[V\left(\rho_{j+1}\right)-V\left(\rho_{j}\right)\right]+a \rho_{0}^{2} \lambda\left[V^{\prime}\left(\rho_{j+1}\right) \tau \partial_{t} \rho_{j+1}-V^{\prime}\left(\rho_{j}(t)\right) \tau \partial_{t} \rho_{j}\right]+a \partial_{t} \rho_{j} \\
-a \gamma\left|\rho_{0}^{2} V^{\prime}\left(\rho_{0}\right)\right|\left(\rho_{j+1}-2 \rho_{j}+\rho_{j-1}\right)-\gamma\left|\rho_{0}^{2} V^{\prime}\left(\rho_{0}\right)\right|\left(\partial_{t} \rho_{j+1}-2 \partial_{t} \rho_{j}+\partial_{t} \rho_{j-1}\right)=0
\end{gathered}
$$

The optimal velocity function $V(\rho)$ is the same as that in Nagatani's model [28]:

$$
V(\rho)=\tanh \left(\frac{2}{\rho_{0}}-\frac{\rho}{\rho_{0}^{2}}-\frac{1}{\rho_{c}}\right)+\tanh \left(\frac{1}{\rho_{c}}\right) .
$$

\section{Linear Stability Analysis}

The objective of stability analysis is to identify the evolution process of the traffic flow with added disturbances. In this section, the linear stability approach is applied to analyze the self-anticipation effect on the two-lane vehicular system.
Suppose that the initial traffic flow keeps constants density $\rho_{0}$ and optimal velocity $V\left(\rho_{0}\right)$. Then, the steady solution of the two-lane vehicular system is

$$
\rho_{j}(t)=\rho_{0}, v_{j}=V\left(\rho_{0}\right) .
$$

Combining small fluctuations $y_{j}(t)$ with equation (10) yields

$$
\rho_{j}(t)=\rho_{0}+y_{j}(t) .
$$

Substituting (11) into (8) and linearizing it, one can deduce

$$
\begin{aligned}
& \partial_{t}^{2} y_{j}+a \rho_{0}^{2} V^{\prime}\left(\rho_{0}\right)\left(y_{j+1}-y_{j}\right)+a \rho_{0}^{2} \lambda \tau V^{\prime}\left(\rho_{0}\right)\left(\partial_{t} y_{j+1}-\partial_{t} y_{j}\right)+a \partial_{t} y_{j} \\
& \quad-a \gamma\left|\rho_{0}^{2} V^{\prime}\left(\rho_{0}\right)\right|\left(y_{j+1}-2 y_{j}+y_{j-1}\right)-\gamma\left|\rho_{0}^{2} V^{\prime}\left(\rho_{0}\right)\right|\left(\partial_{t} y_{j+1}-2 \partial_{t} y_{j}+\partial_{t} y_{j-1}\right)=0,
\end{aligned}
$$


where $V^{\prime}\left(\rho_{0}\right)=d V(\rho) /\left.d \rho\right|_{\rho=\rho_{0}}$. Let $y_{j}(t)=\exp (i k j+z t)$. Substituting the expanding terms of $y_{j}(t)$ into equation (8), one can get the simplified equation

$$
\begin{array}{r}
z^{2}+\left[a \rho_{0}^{2} \lambda \tau V^{\prime}\left(\rho_{0}\right)\left(e^{i k}-1\right)+a-\gamma\left|\rho_{0}^{2} V^{\prime}\left(\rho_{0}\right)\right|\left(e^{i k}-2+e^{-i k}\right)\right] z \\
+a \rho_{0}^{2} V^{\prime}\left(\rho_{0}\right)\left(e^{i k}-1\right)-a \gamma\left|\rho_{0}^{2} V^{\prime}\left(\rho_{0}\right)\right|\left(e^{i k}-2+e^{-i k}\right)=0 .
\end{array}
$$

Expanding the parameter $z$ to $z=z_{1}(i k)+z_{2}(i k)^{2}+\cdots$ and substituting it into equation (13), we can retain the firstorder and second-order terms of $i k$, as

$$
\begin{aligned}
& z_{1}=-\rho_{0}^{2} V^{\prime}\left(\rho_{0}\right), \\
& z_{2}=\frac{1}{2 a}\left(-2 z_{1}^{2}-a \rho_{0}^{2} V^{\prime}\left(\rho_{0}\right)-2 a \gamma \rho_{0}^{2} V^{\prime}\left(\rho_{0}\right)-2 a z_{1} \lambda \tau \rho_{0}^{2} V^{\prime}\left(\rho_{0}\right)\right) .
\end{aligned}
$$

When $z_{2}$ is negative, the steady-state solution of the distribution uniformly inclines to be unstable. On the contrary, it remains to be stable when $z_{2}$ is positive. Thus, the neutral stability condition is derived:

$$
a=-\frac{2 \rho_{0}^{2} V^{\prime}\left(\rho_{0}\right)}{1+2 \gamma-2 \lambda \tau \rho_{0}^{2} V^{\prime}\left(\rho_{0}\right)} .
$$

Hence, the stable condition of the homogeneous traffic flow can be obtained:

$$
a>-\frac{2 \rho_{0}^{2} V^{\prime}\left(\rho_{0}\right)}{1+2 \gamma-2 \lambda \tau \rho_{0}^{2} V^{\prime}\left(\rho_{0}\right)} .
$$

Note that the stable condition of Peng's model [35] is

$$
a>-\frac{3 \rho_{0}^{2} V^{\prime}\left(\rho_{0}\right)}{1+2(\lambda+\gamma)} .
$$

Set $\gamma=0.1$ and $\lambda=0.2$ in both equations (16) and (17). Let $\tau=0.2$. As shown in Figure 2, solid lines represent the neutral stability curves. The phase diagram is divided into two regions with the neutral stability curve: the stable regions and the unstable regions. Then, the stable areas under the new lattice model and Peng's lattice model are both described in Figure 2. It is obvious that the stable area of our model with self-anticipation is larger than that of Peng's lattice model even with a small parameter $\tau$.

As shown in equation (16), we apply the lane-changing coefficient $\gamma$, the anticipation time $\tau$, and the anticipated response coefficient $\lambda$ into the lattice model, which effectively smooths traffic congestion.

\section{Nonlinear Stability Analysis}

In order to analyze the nonlinear characteristics of the equation (8) near the critical point, we adopt the reductive perturbation method and define slow variable $T$, space slow variable $X$, and density $\rho_{j}$ as

$$
\begin{array}{r}
X=\varepsilon(j+b t), T=\varepsilon^{3} t, \quad \rho_{j}=\rho_{c}+\varepsilon R(X, T), \\
0<\varepsilon \ll 1 .
\end{array}
$$

Substitute (18) into (8) and keep the Taylor series expansion of the resulting formula under fifth order of $\varepsilon$. Then, one gets the following equation:

$$
\begin{gathered}
\varepsilon^{2} q_{1} \partial_{X} R+\varepsilon^{3} q_{2} \partial_{X}^{2} R+\varepsilon^{4}\left(\partial_{T} R+q_{3} \partial_{X}^{3} R+q_{4} \partial_{X} R^{3}\right) \\
+\varepsilon^{5}\left(q_{5} \partial_{T} \partial_{X} R+q_{6} \partial_{X}^{4} R+q_{7} \partial_{X}^{2} R^{3}\right)=0 .
\end{gathered}
$$

The coefficients $q_{i}(i=1,2, \ldots, 7)$ are demonstrated in Table 1.

Let $a_{c}=a\left(1+\varepsilon^{2}\right), \quad m=\rho_{0}^{2} V^{\prime}\left(\rho_{0}\right)$, and $b=-m$. The second term and the third term of $\varepsilon$ can be eliminated from equation (19). Then, one can obtain the simplified equation

$$
\varepsilon^{4}\left[\partial_{T} R-g_{1} \partial_{X}^{3} R+g_{2} \partial_{X} R^{3}\right]+\varepsilon^{5}\left[g_{3} \partial_{X}^{2} R+g_{4} \partial_{X}^{4} R+g_{5} \partial_{X}^{2} R^{3}\right]=0,
$$

where

$$
\begin{aligned}
g_{1}= & -m\left(a_{c}-3 m\left(2 \gamma+a_{c} \lambda \tau\right)\right) / 6 a_{c}, \\
g_{2}= & 1 / 6 V^{\prime \prime \prime} \rho_{c}^{2}, \\
g_{3}= & 1 / 2 m(-1-2 \gamma+2 \lambda \tau m), \\
g_{4}= & 1 / 24 a_{c}^{2}\left(m \left(a_{c}^{2}(1+2 \gamma)-8 a_{c} m\left(-1+a_{c} \lambda \tau\right)+12 m^{2}\right.\right. \\
& \left.\left.\left(-2+a_{c} \lambda \tau\right)\left(2 \gamma+a_{c} \lambda \tau\right)\right)\right), \\
g_{5}= & -1 / 12 a_{c}\left(\rho_{c}^{2}\left(-a_{c} V^{\prime \prime \prime}+2 m\left(-2+a_{c} \lambda \tau\right)+a_{c} \lambda \tau V^{\prime \prime \prime}\right)\right) .
\end{aligned}
$$

For the sake of computing the propagation velocity, the following condition must be satisfied:

$$
\left(R_{0}^{\prime}, M\left[R_{0}^{\prime}\right]\right)=\int_{-\infty}^{+\infty} d X^{\prime} R_{0}^{\prime} M\left[R_{0}^{\prime}\right]=0 .
$$




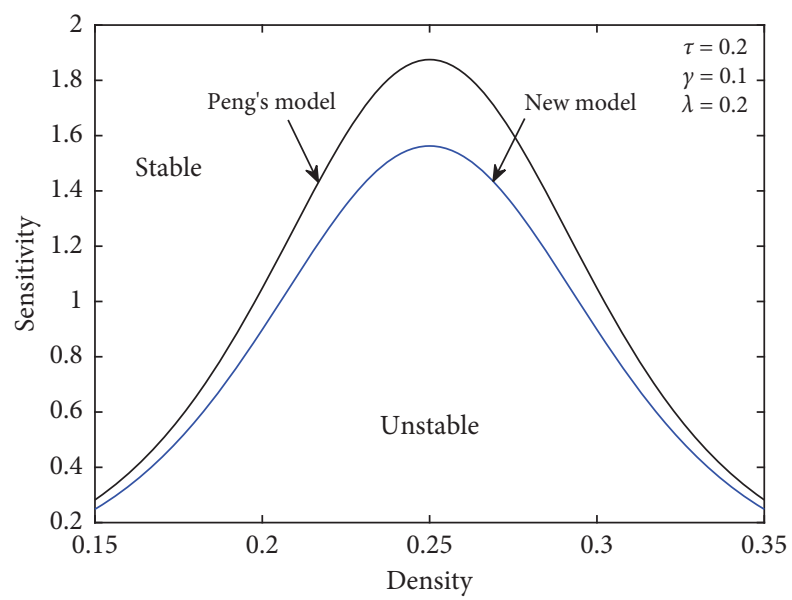

Figure 2: Phase diagram in parameter space $(\rho, a)$ with the new model and Peng's model.

TABLE 1: The coefficient $q_{i}$ of the model.

\begin{tabular}{lr}
\hline$q_{1}$ & $q_{2}$ \\
\hline$b+m$ & $b^{2} / a+\gamma m+1 / 2 m+b \lambda \tau m$ \\
$q_{3} / a \gamma m+1 / 6 m+1 / 2 b \lambda \tau m$ & $q_{4}$ \\
$q_{5}$ & $1 / 6 \rho_{c}^{2} V^{\prime \prime \prime}$ \\
$2 b / a+\lambda \tau m$ & $q_{6}$ \\
$q_{7}$ & $1 / 12 \gamma m+1 / 24 m+1 / 6 b \lambda \tau m$ \\
$1 / 12 \rho_{c}^{2} V^{\prime \prime \prime}+1 / 6 b \lambda \tau m$ & \\
\hline
\end{tabular}

With the selected velocity $c=5 g_{2} g_{3} / 2 g_{2} g_{4}-3 g_{1} g_{5}$, the kink-antikink soliton solution can be expressed by

$$
\rho_{j}=\rho_{c}+\varepsilon \sqrt{\frac{g_{1} c}{g_{2}}} \tanh \left[\sqrt{\frac{c}{2}}\left(X-c g_{1} T\right)\right],
$$

and the amplitude of the derived solution is

$$
A=\sqrt{\frac{g_{1}}{g_{2}} \varepsilon^{2} c}
$$

Both the freely moving phase and the congested phase are contained into the co-existing phase in the kink-antikink solution, which is, respectively, described by $\rho_{j}=\rho_{c}-A$ and $\rho_{j}=\rho_{c}+A$ in the space $(\rho, a)$. In each pattern of Figure 3, solid lines and dashed lines represent the neutral stability curves and the co-existing curves, respectively. With an increasing anticipation time $\tau$ in Figures 3(a) and 3(b), it is clear that the apex of curves $\left(\rho_{c}, a_{c}\right)$ decreases no matter whether lane changing occurs or not. This indicates that traffic congestions can be alleviated more efficiently with the increasing value of $\tau$ in both cases. Comparing Figures 3(a) and $3(b)$, the stability region with lane changing $(\gamma=0.1)$ is significantly larger than that without lane changing $(\gamma=0)$. Moreover, the corresponding stable region expands gradually with the increasing value of $\gamma$ when $\tau=0.4$ as shown in Figure 3(c). This phenomenon demonstrates that the lanechanging behavior could be favorable to the stability enhancement of traffic flow. In summary, the SCDE effect and the lane-changing behavior both play positive roles in the improvement of the traffic flow stability.

\section{Numerical Simulations}

In this section, numerical simulations are provided to demonstrate the effect of driver's self-anticipation in twolane traffic flow with linear as well as nonlinear stability analysis. The initial densities are adopted with the periodic boundary conditions as follows:

$$
\rho_{j}(1)=\rho_{j}(0)=\left\{\begin{array}{l}
\rho_{0} ; j \neq \frac{N}{2}, \frac{N}{2}+1 \\
\rho_{0}+\eta ; j=\frac{N}{2} \\
\rho_{0}-\eta ; j=\frac{N}{2}+1
\end{array},\right.
$$

where the initial disturbance $\eta$ is 0.01 and total amount of lattices $N$ is 200. Following Reference [35], the related parameters with the SCDE effect are chosen as $a=1.5$, $\lambda=0.2$, and $\rho_{0}=\rho_{c}=0.25$.

The evolution of density waves is exhibited in Figures 4-7 with an anticipation time variable $\tau$. Figures 4-7, respectively, correlate with the no lane-changing case $(\gamma=0)$ and the lanechanging case $(\gamma=0.1)$. 


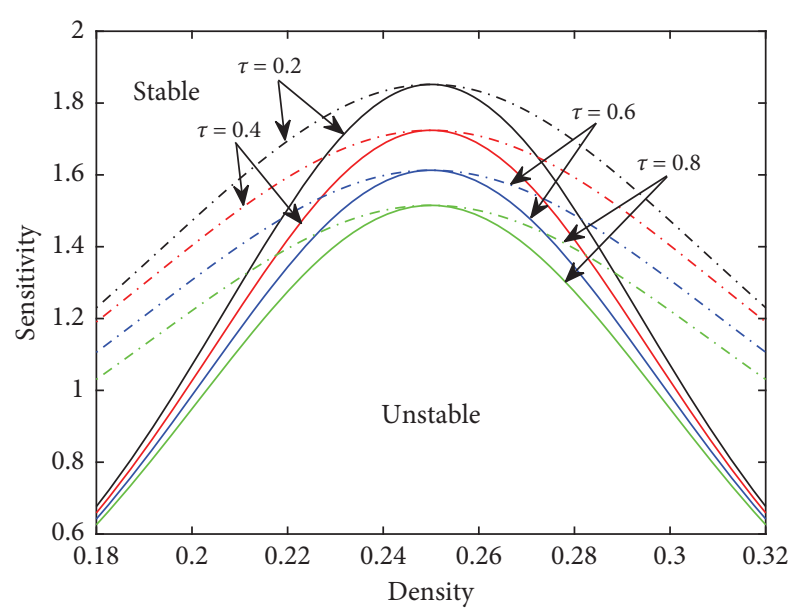

(a)

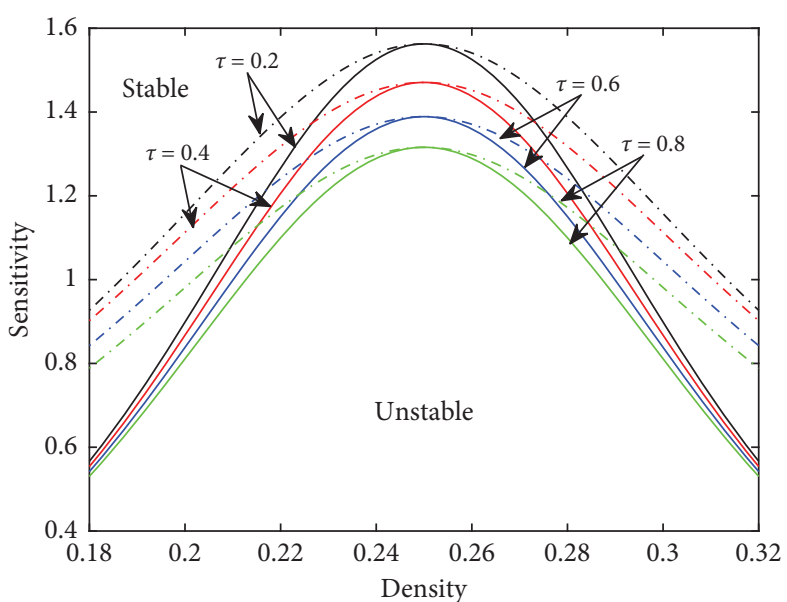

(b)

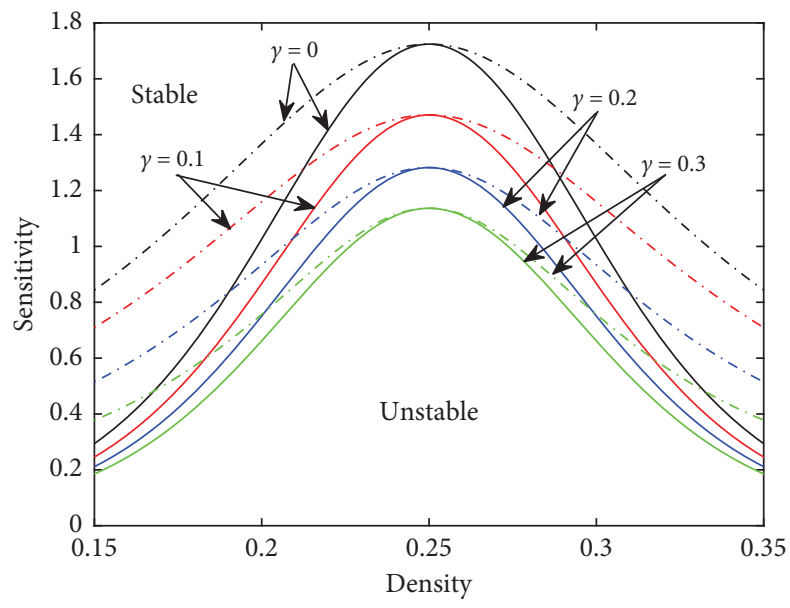

(c)

Figure 3: Phase diagram in parameter space $(\rho, a)$ with different values of parameters $\tau$ and $\gamma$ when $\lambda=0.2$. (a) $\gamma=0$. (b) $\gamma=0.1$. (c) $\tau=0.4$.

Case 1. No lane changing $(\gamma=0)$.

Since the given parameters in Figures 4(a)-4(c) do not satisfy the stable condition equation (16), the added disturbance will cause the kink-antikink density waves. Meanwhile, Figures 4(a)-4(d) and Figures 5(a)-5(d) reveal that the amplitude of density waves decreases with the rising value of $\tau(\tau=0.2,0.4,0.6$, and 0.8 , respectively) and the traffic congestion does not disappear until $\tau=0.8$. In other words, the driver's self-anticipation can efficiently suppress the traffic jam even without lane-changing behavior.

Case 2. Lane changing $(\gamma=0.1)$.

Figures 6 and 7 demonstrate the simulation results under the case of lane changing with the same anticipation time variable $\tau$ defined in Figures 4 and 5. The relationship between density waves and the variable $\tau$ in Figure 6 is similar to that in Figure 4. Moreover, the amplitudes of density waves in Figure 7 are smaller than their counterparts in Figure 5. This phenomenon indicates that appropriate lane changing could further promote the stabilization of traffic flow which incorporates driver's self-anticipation.

Figures $8(\mathrm{a})$ and $8(\mathrm{~b})$ show the phase space of density difference $\rho(t)-\rho(t-1)$ against $\rho(t)$ for $t=10000-12000$ without and with lane changing under different $\tau$, respectively. The pattern exhibits the behavior characteristic of chaos. In Figure 8(a), with increasing $\tau$, the points are denser and the amplitude of the limit cycle is smaller. When $\tau \geq 0.8$, phase states converge to a stable point, which means that the density difference is zero, i.e., the traffic system without lane changing is stable. In Figure 8(b), the variation characteristic of the limit cycle with lane changing is similar to the one without lane changing. Although the cycles in Figure 8(b) are more irregular than those in Figure 8(a) under the same $\tau$, the traffic system is easier to be stable. Note that when $\tau \geq 0.6$, the traffic flow becomes stable in Figure 8(b), but the traffic flow remains unstable until $\tau \geq 0.8$ in Figure 8(a). It also validates that the self-anticipation current difference effect with lane changing can effectively relieve traffic congestion. 


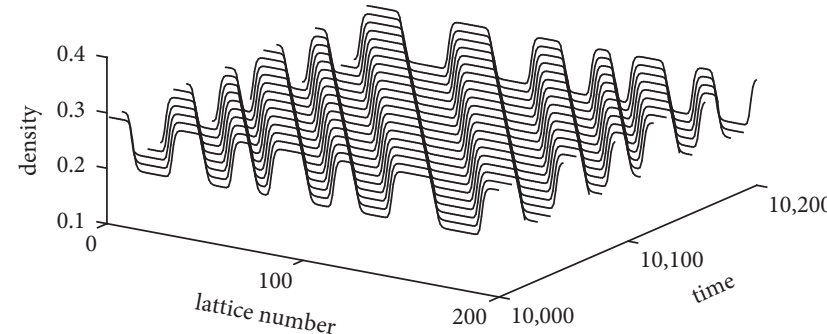

(a)

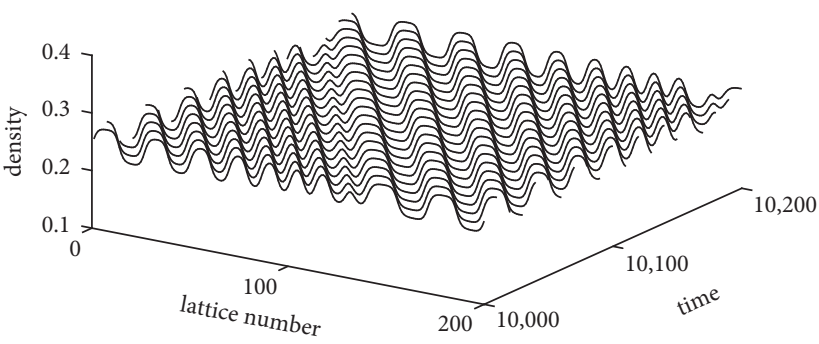

(c)

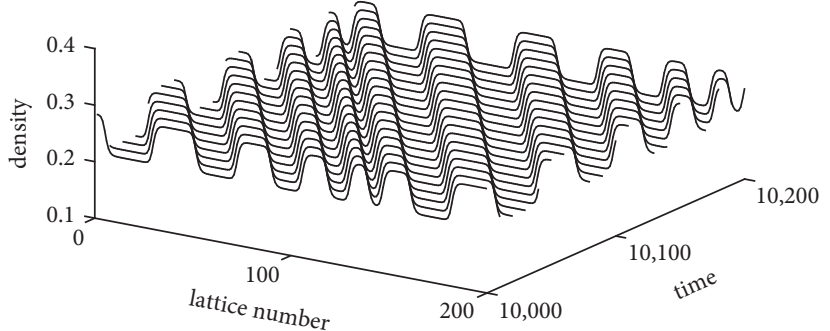

(b)

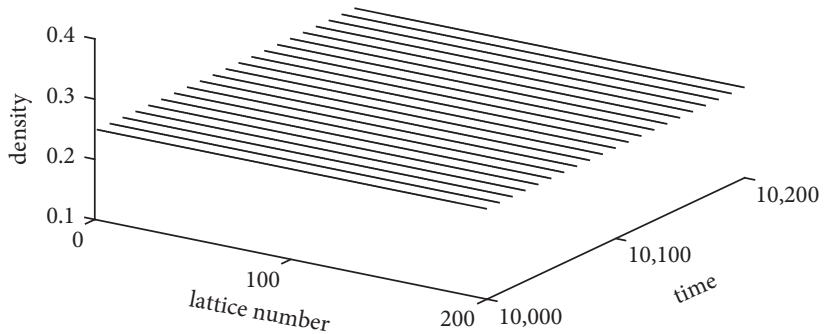

(d)

Figure 4: Spatiotemporal evolutions of density (for time $t=10000-10200 \mathrm{~s}$ ) when $\gamma=0, \lambda=0.2$, and $a=1.5$ for different values of $\tau$. (a) $\tau=0.2$. (b) $\tau=0.4$. (c) $\tau=0.6$. (d) $\tau=0.8$.

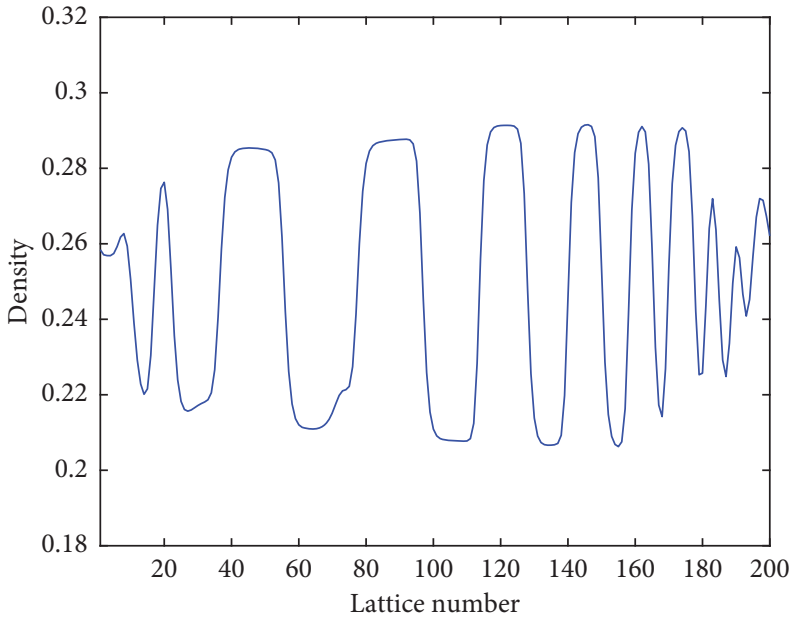

(a)

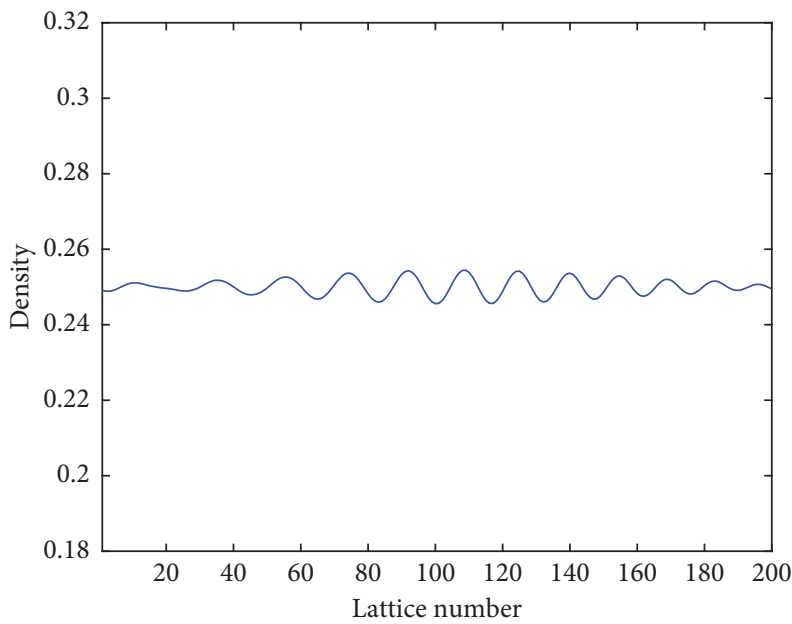

(c)

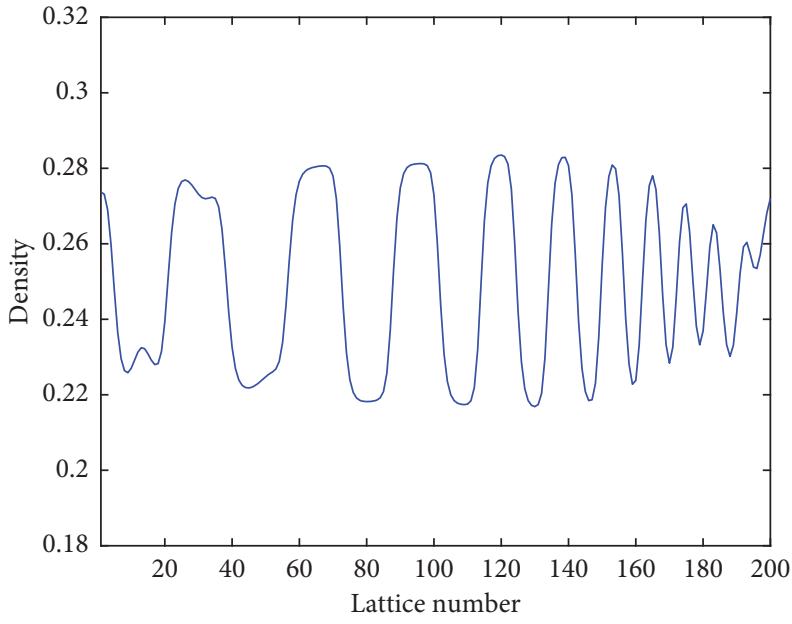

(b)

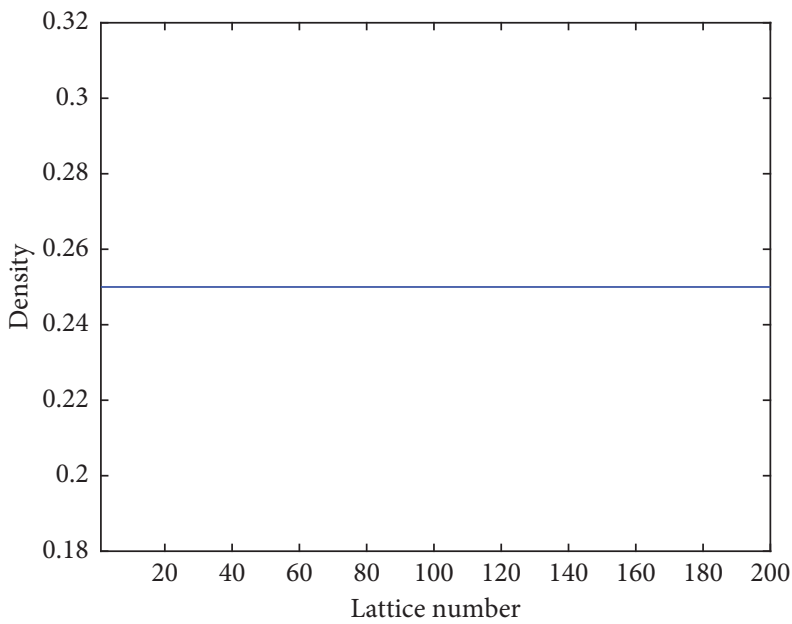

(d)

Figure 5: Density profile of the density wave at time $t=10200 \mathrm{~s}$ for (a) $\tau=0.2$, (b) $\tau=0.4$, (c) $\tau=0.6$, and (d) $\tau=0.8$ when $\gamma=0$. 


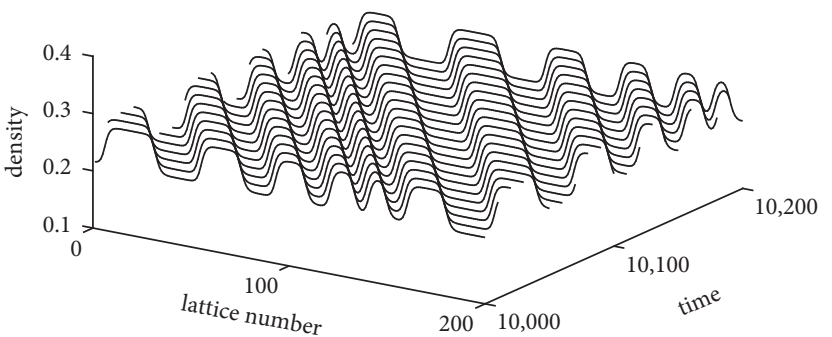

(a)

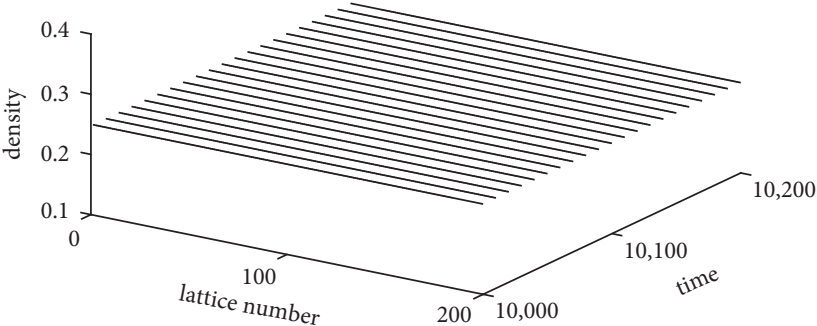

(c)

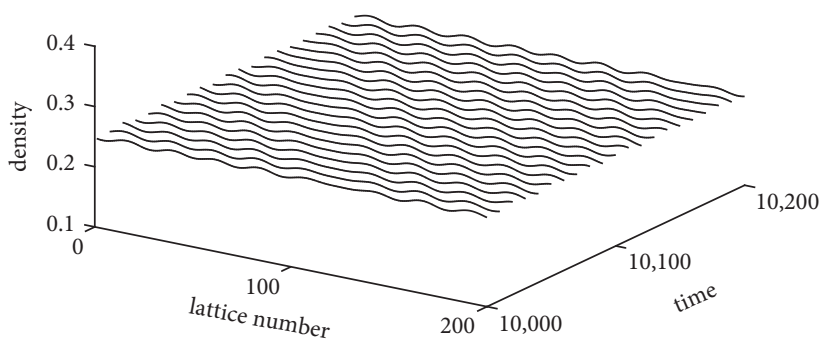

(b)

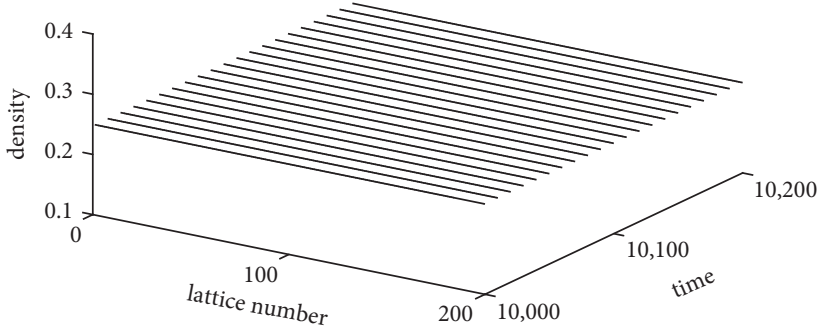

(d)

FIGURE 6: Spatiotemporal evolutions of density (for time $t=10000-10200 \mathrm{~s}$ ) when $\gamma=0.1, \lambda=0.2$, and $a=1.5$ for different values of $\tau$.

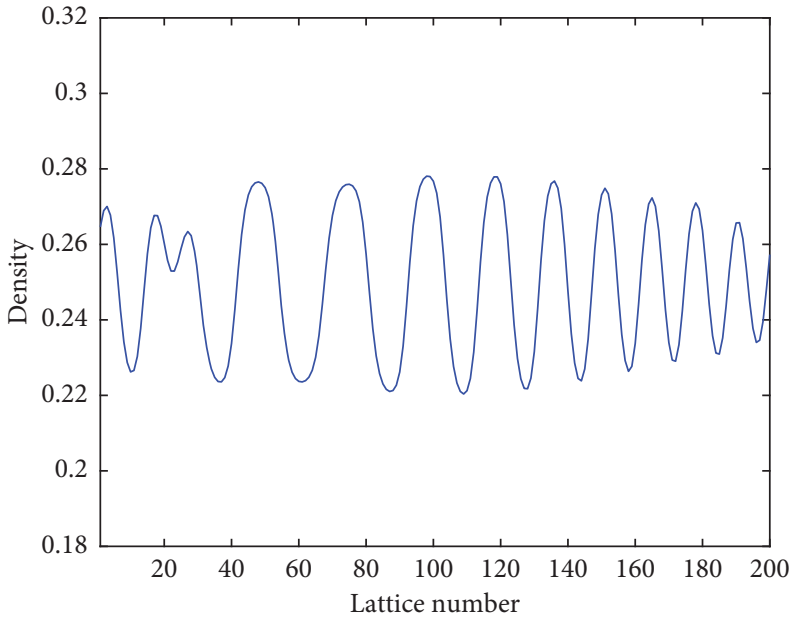

(a)

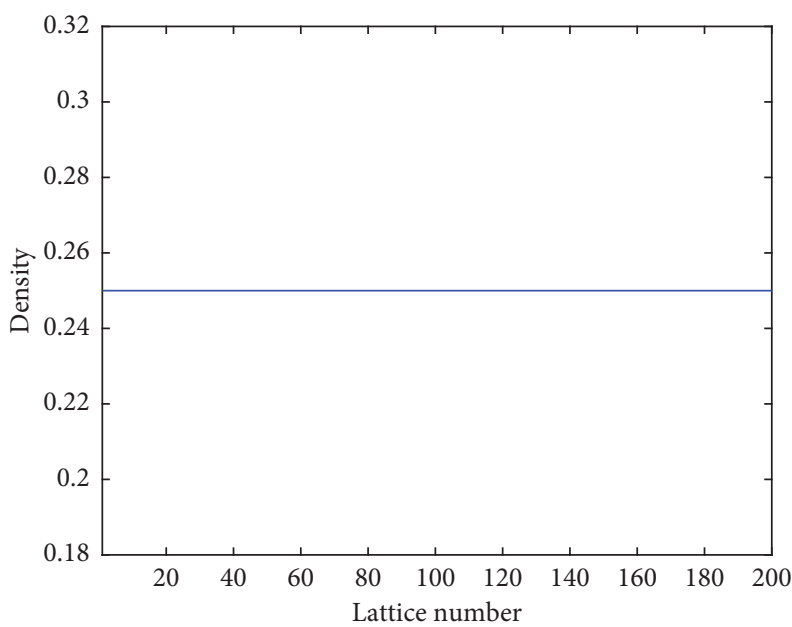

(c)

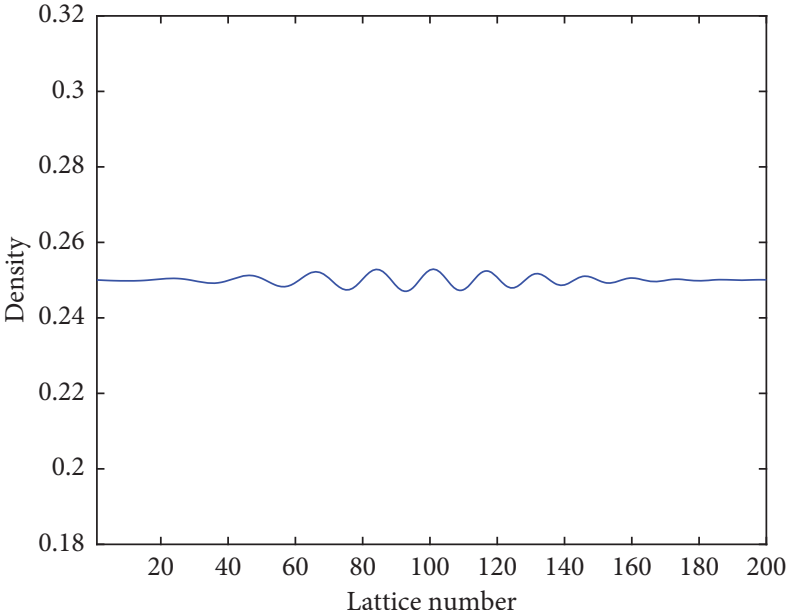

(b)

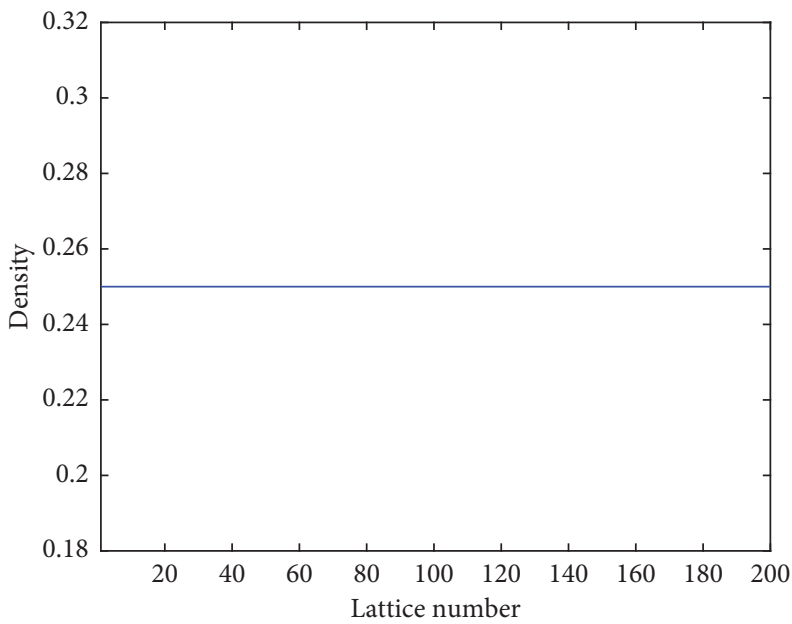

(d)

Figure 7: Density profile of the density wave at time $t=10200 \mathrm{~s}$ for (a) $\tau=0.2$, (b) $\tau=0.4$, (c) $\tau=0.6$, and (d) $\tau=0.8$ when $\gamma=0.1$. 


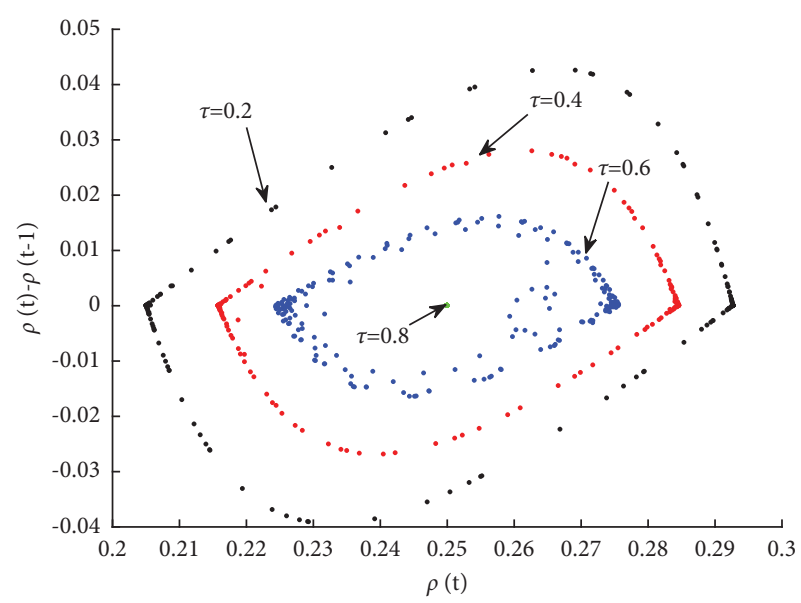

(a)

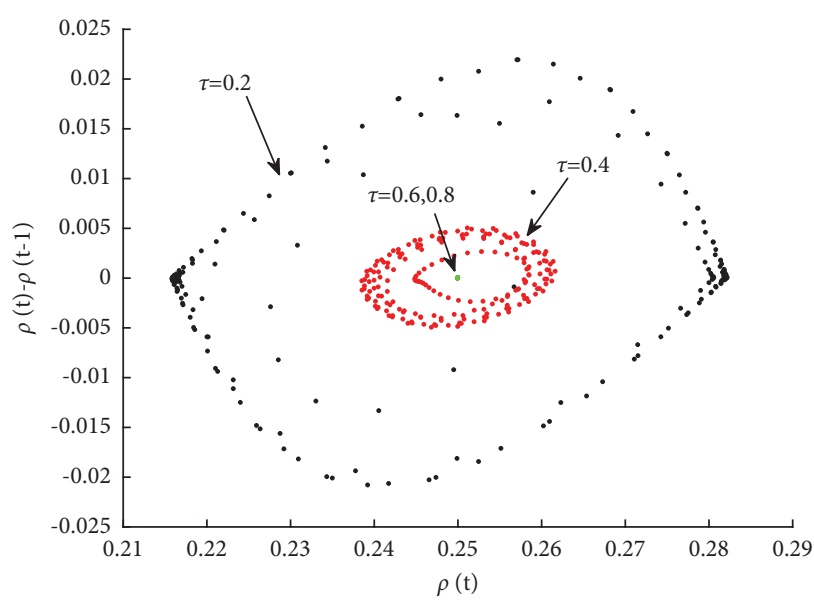

(b)

Figure 8: Density difference (a) $\rho(t)-\rho(t-1)$ against density (b) $\rho(t)$ corresponding to the panels in Figures 5 and 7 , respectively.

\section{Conclusion}

In this paper, a novel lattice hydrodynamic model is constructed to explore the anticipation effect concerning the SCDE for a two-lane traffic system. The neutral stability condition and the solution of the $\mathrm{mKdV}$ equation are deduced to describe traffic jams. The results of theoretical analysis demonstrate that both the anticipation time $\tau$ and the coefficient of lane-changing behaviors $\gamma$ are major factors for the enhancement of traffic flow stability. The numerical simulation results validate the theoretical analysis, which indicates that the self-anticipation current difference effect can push forward an immense influence on the stability of traffic flow. Future work will focus on solving the traffic congestion on message propagation mechanism, the characteristics of drivers, the vehicular emergency warning system, and the automation for longitudinal driving.

\section{Data Availability}

The data used to support the findings of this study are available from the corresponding author upon request.

\section{Conflicts of Interest}

The authors declare that there are no conflicts of interest regarding the publication of this paper.

\section{Acknowledgments}

This study was supported in part by the National Natural Science Foundation of China under grant nos. 61903203 and 62073180 and Nantong Science \& Technology Research Plan (no. JC2021133).

\section{References}

[1] Y. Zhang, M. Zhao, D. Sun, S. h. Wang, S. Huang, and D. Chen, "Analysis of mixed traffic with connected and nonconnected vehicles based on lattice hydrodynamic model,"
Communications in Nonlinear Science and Numerical Simulation, vol. 94, Article ID 105541, 2021.

[2] C. Zhu, S. Zhong, and S. Ma, "Two-lane lattice hydrodynamic model considering the empirical lane-changing rate," Communications in Nonlinear Science and Numerical Simulation, vol. 73, pp. 229-243, 2019.

[3] T. Tang, H. Huang, S. Wong, and R. Jiang, "Lane changing analysis for two-lane traffic flow," Acta Mechanica Sinica, vol. 23, no. 49-54, 2007.

[4] G. Peng, K. Bai, and H. Kuang, "Feedback control caused by honk effect incorporating the driver's characteristics in lattice hydrodynamic model," Physica A: Statistical Mechanics and Its Applications, vol. 526, Article ID 121064, 2019.

[5] P. Redhu and A. K. Gupta, "Delayed-feedback control in a lattice hydrodynamic model," Communications in Nonlinear Science and Numerical Simulation, vol. 27, no. 1-3, pp. 263-270, 2015.

[6] H. Zhao, H. Yue, T. Gu, and W. Li, "Cps-based reliability enhancement mechanism for vehicular emergency warning system," International Journal of Intelligent Transportation Systems Research, vol. 17, no. 3, pp. 232-241, 2019.

[7] H. Zhao, H. Yue, T. Gu, C. Li, and D. Zhou, "Low delay and seamless connectivity-based message propagation mechanism for vanet of vcps," Wireless Personal Communications, vol. 118, no. 4, pp. 3385-3402, 2021.

[8] R. Cheng, H. Ge, and J. Wang, "An extended macro traffic flow model accounting for multiple optimal velocity functions with different probabilities," Physics Letters A, vol. 381, no. 32, pp. 2608-2620, 2017.

[9] H. Liu, P. Zheng, K. Zhu, and H. Ge, "KdV-Burgers equation in the modified continuum model considering anticipation effect," Physica A: Statistical Mechanics and Its Applications, vol. 438, pp. 26-31, 2015.

[10] R. Cheng, H. Ge, and J. Wang, "Kdv-burgers equation in a new continuum model based on full velocity difference model considering anticipation effect," Physica A: Statistical Mechanics and Its Applications, vol. 481, no. 52-59, 2017.

[11] L. Sun, A. Jafaripournimchahi, A. Kornhauser, and W. Hu, "A new higher-order viscous continuum traffic flow model considering driver memory in the era of autonomous and connected vehicles," Physica A: Statistical Mechanics and Its Applications, vol. 547, Article ID 123829, 2020. 
[12] W.-X. Zhu and L.-D. Zhang, "Analysis of car-following model with cascade compensation strategy," Physica A: Statistical Mechanics and Its Applications, vol. 449, pp. 265-274, 2016.

[13] M. Ma, G. Ma, and S. Liang, "Density waves in car-following model for autonomous vehicles with backward looking effect," Applied Mathematical Modelling, vol. 94, no. 1-12, 2021.

[14] T. Tang, H. Huang, S. C. Wong, and R. Jiang, "A car-following model with the anticipation effect of potential lane changing," Acta Mechanica Sinica, vol. 24, no. 4, pp. 399-407, 2008.

[15] T. Tang, W. Shi, H. Shang, and Y. Wang, "A new car-following model with consideration of inter-vehicle communication," Nonlinear Dynamics, vol. 76, no. 4, pp. 2017-2023, 2014.

[16] H. Kuang, Z.-P. Xu, X.-L. Li, and S.-M. Lo, "An extended carfollowing model accounting for the honk effect and numerical tests," Nonlinear Dynamics, vol. 87, no. 1, pp. 149-157, 2016.

[17] W.-X. Zhu and Li-D. Zhang, "A new car-following model for autonomous vehicles flow with mean expected velocity field," Physica A: Statistical Mechanics and Its Applications, vol. 492, pp. 2154-2165, 2018.

[18] H. Kuang, Z.-P. Xu, X.-L. Li, and S.-M. Lo, "An extended carfollowing model accounting for the average headway effect in intelligent transportation system," Physica A: Statistical Mechanics and Its Applications, vol. 471, pp. 778-787, 2017.

[19] Z. Hongzhuan, Q. Zhao, W. Chen, T. Shi, W. Gu, and S. Li, "Stability analysis of an improved car-following model accounting for the driver's characteristics and automation - sciencedirect," Physica A: Statistical Mechanics and Its Applications, vol. 526, Article ID 120990, 2019.

[20] T.-Q. Tang, Y.-X. Rui, J. Zhang, and H.-Y. Shang, "A cellular automation model accounting for bicycle's group behavior," Physica A: Statistical Mechanics and Its Applications, vol. 492, pp. 1782-1797, 2018.

[21] M. Zamith, R. C. P. Leal-Toledo, E. Clua, E. M. Toledo, and G. V. P. D. Magalhães, "A new stochastic cellular automata model for traffic flow simulation with drivers' behavior prediction," Journal of Computational Science, vol. 9, pp. 51-56, 2015.

[22] C. Zhai and W. Wu, "Designing continuous delay feedback control for lattice hydrodynamic model under cyber-attacks and connected vehicle environment," Communications in Nonlinear Science and Numerical Simulation, vol. 95, Article ID 105667, 2021.

[23] G. Zhang and G.-H. Peng, "Research on the stabilization effect of continuous self-delayed traffic flux in macro traffic modeling," Physica A: Statistical Mechanics and Its Applications, vol. 526, Article ID 121012, 2019.

[24] G. Zhang, Y. Zhang, D.-B. Pan, and R.-J. Huang, "Study on the continuous delayed optimal flow on traffic stability in a new macro traffic model," Physica A: Statistical Mechanics and Its Applications, vol. 534, Article ID 122029, 2019.

[25] H. Z. Zhao, D. Xia, S. Yang, and G. Peng, "The delayed-time effect of traffic flux on traffic stability for two-lane freeway," Physica A: Statistical Mechanics and Its Applications, vol. 540, Article ID 123066, 2019.

[26] G. Peng, "A new lattice model of traffic flow with the consideration of individual difference of anticipation driving behavior," Communications in Nonlinear Science and Numerical Simulation, vol. 18, no. 10, pp. 2801-2806, 2013.

[27] T. Nagatani, "Modified KdV equation for jamming transition in the continuum models of traffic," Physica A: Statistical Mechanics and Its Applications, vol. 261, no. 3-4, pp. 599-607, 1998.

[28] T. Nagatani, "Jamming transitions and the modified Korteweg-de Vries equation in a two-lane traffic flow," Physica A:
Statistical Mechanics and Its Applications, vol. 265, no. 1-2, pp. 297-310, 1999.

[29] G. Peng, S. Yang, D. Xia, and X. Li, "A novel lattice hydrodynamic model considering the optimal estimation of flux difference effect on two-lane highway," Physica A: Statistical Mechanics and Its Applications, vol. 506, pp. 929-937, 2018.

[30] S. Qin, Z. He, and R. Cheng, "An extended lattice hydrodynamic model based on control theory considering the memory effect of flux difference," Physica A: Statistical Mechanics and Its Applications, vol. 509, pp. 809-816, 2018.

[31] D.-H. Sun, G. Zhang, M. Zhao, S.-L. Cheng, and J.-D. Cao, "Stability analysis of feedforward anticipation optimal flux difference in traffic lattice hydrodynamic theory," Communications in Nonlinear Science and Numerical Simulation, vol. 56, pp. 287-295, 2018.

[32] T. Wang, Z. Gao, J. Zhang, and X. Zhao, "A new lattice hydrodynamic model for two-lane traffic with the consideration of density difference effect," Nonlinear Dynamics, vol. 75, no. 27-34, 2014.

[33] T. Wang, Z. Gao, W. Zhang, J. Zhang, and S. Li, "Phase transitions in the two-lane density difference lattice hydrodynamic model of traffic flow," Nonlinear Dynamics, vol. 77, no. 3, pp. 635-642, 2014.

[34] C. Liu, Y. He, and G. Peng, "The impact of the density difference memory integral on traffic stability in two-lane lattice hydrodynamic model," Physica A: Statistical Mechanics and Its Applications, vol. 532, Article ID 121750, 2019.

[35] G. Peng, "A new lattice model of two-lane traffic flow with the consideration of optimal current difference," Communications in Nonlinear Science and Numerical Simulation, vol. 18, no. 3, pp. 559-566, 2013.

[36] G. Peng, W. Lu, and H. He, "Impact of the traffic interruption probability of optimal current on traffic congestion in lattice model," Physica A: Statistical Mechanics and Its Applications, vol. 425, pp. 27-33, 2015.

[37] S. Sharma, "Effect of driver's anticipation in a new two-lane lattice model with the consideration of optimal current difference," Nonlinear Dynamics, vol. 81, no. 1-2, pp. 991-1003, 2015.

[38] A. K. Gupta and P. Redhu, “Analyses of driver's anticipation effect in sensing relative flux in a new lattice model for two-lane traffic system," Physica A: Statistical Mechanics and Its Applications, vol. 392, no. 22, pp. 5622-5632, 2013.

[39] A. K. Gupta and P. Redhu, "Analyses of the driver's anticipation effect in a new lattice hydrodynamic traffic flow model with passing," Nonlinear Dynamics, vol. 76, no. 2, pp. 1001-1011, 2014.

[40] G. Zhang, D.-H. Sun, H. Liu, and D. Chen, "Stability analysis of a new lattice hydrodynamic model by considering lattice's self-anticipative density effect," Physica A: Statistical Mechanics and Its Applications, vol. 486, pp. 806-813, 2017.

[41] T. Wang, R. Zang, K. Xu, and J. Zhang, "Analysis of predictive effect on lattice hydrodynamic traffic flow model," Physica A: Statistical Mechanics and Its Applications, vol. 526, Article ID 120711, 2019. 